Enferm Bras 2019;18(1):75-84

http://dx.doi.org/10.33233/eb.v18i1.2480

\title{
ARTIGO ORIGINAL \\ A atenção primária em saúde como fonte de apoio social a gestantes adolescentes
}

Pamela Nery do Lago*, Albertina Antonielly Sydney de Sousa**, Dafne Paiva Rodrigues***,

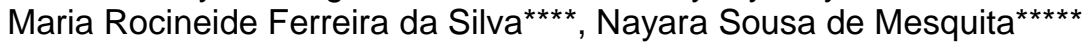

*Enfermeira do Hospital das Clínicas da Universidade Federal de Minas Gerais (HCUFMG/EBSERH), ${ }^{* *}$ Docente do curso de Graduação em Enfermagem do Centro Universitário Estácio, ${ }^{* *}$ Docente do curso de Graduação em Enfermagem e do Programa de PósGraduação Cuidados Clínicos em Enfermagem e Saúde (PPCCLIS) da UECE, ${ }^{* * * *}$ Professora Adjunta do Curso de Enfermagem e da Pós-Graduação nos Programas de Saúde Coletiva, Cuidados Clínicos em Enfermagem e Saúde, Saúde Coletiva e Mestrado Profissional em Saúde da Família da UECE, ${ }^{\star \star \star \star \star} E$ Enfermeira do Instituto Federal de Ciência, Tecnologia e Educação (IFCE)

Recebido em 6 de agosto de 2018; aceito em 8 de setembro de 2018.

Endereço para correspondência: Pamela Nery do Lago, Hospital das Clínicas da Universidade Federal de Minas Gerais, Av. Prof. Alfredo Balena, 110, Santa Efigênia, 30130100 Belo Horizonte MG, E-mail: pamelabio@yahoo.com.br; Albertina Antonielly Sydney de Sousa: albertina_sousa@hotmail.com; Dafne Paiva Rodrigues: dafne.rodrigues@uece.br; Maria Rocineide Ferreira da Silva: rocineideferreira@gmail.com; Nayara Sousa de Mesquita: nayara_sousadm@hotmail.com

\section{Resumo}

Objetivou-se investigar a atuação da atenção primária em saúde na rede de apoio social à gestante adolescente. Trata-se de uma pesquisa exploratório-descritiva, qualitativa, realizada com 16 gestantes entre 12 e 18 anos e com enfermeiros, de fevereiro a maio de 2014, em quatro Unidades de Atenção Primária à Saúde da cidade de Fortaleza/CE, além do domicílio das gestantes. Para a coleta dos dados com as gestantes, utilizou-se uma entrevista semiestruturada; para avaliar a atuação do enfermeiro nas consultas de pré-natal, realizou-se a observação sistemática. Da análise dos dados pela técnica de análise de conteúdo, emergiu a categoria $O$ apoio social da Atenção Primária à Saúde na maternidade adolescente. Nenhuma participante possuía vínculo empregatício e algumas relataram abandono dos estudos; mais da metade era casada/amasiada e todas afirmaram ser católicas. A rede de apoio social contribuiu significativamente para as gestantes adolescentes no enfrentamento das adversidades inerentes ao seu processo adaptativo à nova realidade. Contudo, percebeu-se que o pouco apoio social oriundo dos enfermeiros foi um ponto negativo, revelando vínculos frágeis e pouco efetivos na contribuição da adoção do papel materno.

Palavras-chave: gravidez na adolescência, apoio social, atenção primária à saúde, Enfermagem.

\footnotetext{
Abstract

Primary health care as source of social support of pregnant adolescents

This study aimed to investigate the role of primary health care in the network of social support of pregnant adolescents. It is an exploratory-descriptive, qualitative research conducted with 16 pregnant women between 12 and 18 years old and with nurses, from February to May 2014, in four Primary Health Units in the city of Fortaleza/CE, and at adolescents' home. To collect the data with pregnant adolescents, was used a semi-structured interview; to evaluate the work of nurses in prenatal consultation, was done a systematic observation. Data analysis by the content analysis technique, revealed the category Social support by primary health care in the adolescent motherhood. The participants were not employed and some reported school dropout; more than half were married / consensual union and all were catholic. The social support network contributed significantly to the pregnant adolescents in facing adversities inherent in their adaptation process to the new reality. However, it was perceived, as a negative point, the
} 
little social support coming from the nurses, revealing weak and ineffective relationships in the contribution on the maternal role adoption.

Key-words: pregnancy in adolescence, social support, primary health care, Nursing.

\section{Resumen \\ La atención primaria en salud como fuente de apoyo social a gestantes adolescentes}

Se objetivó investigar la actuación de la atención primaria en salud en la red de apoyo social a la gestante adolescente. Se trata de una investigación exploratoria-descriptiva, cualitativa, realizada con 16 gestantes y con enfermeros, de febrero a mayo de 2014, en 04 Unidades de Atención Primaria a la Salud de Fortaleza/CE y en el domicilio de las gestantes. En la recolección de los datos con las gestantes, se utilizó la entrevista semiestructurada; para evaluar la actuación del enfermero en las consultas de prenatal, se realizó la observación sistemática. Del análisis de los datos por la técnica de análisis de contenido, emergió la categoría El apoyo social de la Atención Primaria a la Salud en la maternidad adolescente. Las participantes tenían edades entre 14 y 18 años; sin vínculo laboral y algunas con abandono de los estudios; más de la mitad estaba casada y todas católicas. La red de apoyo social contribuyó a las gestantes adolescentes en el enfrentamiento de las adversidades inherentes al proceso adaptativo. Sin embargo, el poco apoyo social proveniente de los enfermeros fue un punto negativo, revelando vínculos frágiles y poco efectivos en la contribución de la adopción del papel materno.

Palabras-clave: embarazo en adolescencia, apoyo social, atención primaria de salud, Enfermería.

Introdução

A adolescência é uma fase do desenvolvimento humano muito importante para atingir a maturidade biopsicossocial, na qual a sexualidade manifesta-se em novas e surpreendentes necessidades e sensações corporais [1]. Esta se constitui em um momento de descobertas, mudanças e escolhas, envoltas em um turbilhão de hormônios, o que, em conjunto, ocasiona um período de grande instabilidade emocional tanto para a adolescente como para os que a cercam.

Quando uma gestação se apresenta neste cenário, aumentam-se consideravelmente os fatores estressores, principalmente quando a gravidez não é planejada e/ou desejada. A adolescente vê-se mudando da posição de um indivíduo até então cuidado pela família, para outra que a eleva à condição de adulto responsável por cuidar de uma nova vida.

A transição entre a posição de indivíduo que recebe cuidados para a do que oferece cuidados ocorre, geralmente, num contexto de coabitação e dependência sociofamiliar. E a opção pela gravidez encerra o ônus da ilegitimidade social da maternidade precoce, atribuída frequentemente à "irresponsabilidade" adolescente. A forma como a família, companheiro e amigos percebem esse processo, influenciará diretamente na maneira como a adolescente vivenciará este momento [2].

Neste contexto, destaca-se o apoio social como elemento facilitador na vivência da maternidade adolescente. Além de amenizar o sofrimento, o qual pode afetar negativamente a saúde, o apoio social se constitui em um fator de proteção à vida nos aspectos físico, mental e psicoafetivo [3]. O indivíduo passa a ser protagonista da sua vida, recebendo apoio através de suas redes para controlar seu destino, favorecendo a construção da capacidade social e pessoal pela interação com os que fazem parte do seu convívio.

Estudos mostram que, no âmbito da gravidez adolescente, o suporte social influencia positivamente na saúde das jovens mães [3-5]. Apesar do caráter protetor do apoio social, o que a literatura tem mostrado é que as redes sociais de gestantes adolescentes tendem a ser pouco diversificadas, com predominância dos membros da família, muitas vezes marcadas por conflitos, tensões e discussões frequentes [6]. As outras pessoas incluídas nas redes significativas geralmente figuram relações apenas de contato social ou distante, e que não desempenham funções significativas de ajuda no enfrentamento das dificuldades vivenciadas [6].

O enfermeiro surge nesse cenário, não apenas como membro da equipe de saúde que executa suas tarefas assistenciais, durante o pré-natal, parto e pós-parto, mas também como um profissional com competências para ajudar e direcionar a adolescente diante dos conflitos que envolvem a gravidez na adolescência. Torna-se imperativo um agir ético que envolva também a família, suprindo não apenas a necessidade de cuidado biológico como também 
psicossocial; assim, surgem outros profissionais da área da saúde e da educação que podem somar esforços para atender, de forma mais integral, à jovem e sua família [7].

Sobre os conflitos que emergem na família da adolescente, durante e depois do descobrimento da gestação, cabe ressaltar que as ações de enfermagem devem objetivar a subjetividade do cuidado humano e o atendimento global às necessidades de saúde, ou seja, envolver a gestante e sua família, incentivar e permitir a participação dos familiares nos grupos de gestantes e consultas de enfermagem, priorizando também um espaço na agenda da enfermagem para visitas domiciliares ao lar dessa família [7].

Neste contexto, oportuniza-se ao enfermeiro o desenvolvimento de uma consulta prénatal e puerperal qualificada e acolhedora, visando aproximar-se da realidade da gestante adolescente, compreendendo qual a composição de sua rede de apoio e fornecendo subsídios a uma gestação, parto e puerpério mais confortáveis e seguros, configurando-se como outra significativa fonte de apoio social, além da família.

O profissional de enfermagem necessita estabelecer interações efetivas e relações de cuidado embasada na solidariedade, apoio emocional, conforto e calor humano, dada as circunstâncias do processo de pré, trans e pós-parto [8]. Traça-se, desta forma, um paralelo com o que é proposto pelo Pacto pela Vida [9], que traz em seu texto a importância de se garantir o direito da gestante ao acesso a um atendimento digno e de qualidade na gestação/parto e puerpério.

A compreensão da tessitura da rede de apoio social das gestantes adolescentes pode promover um cuidado mais integral, voltado tanto para suas necessidades biológicas como para as necessidades psicossociais que as cercam neste ciclo de vida tão particular. Desta forma, o enfermeiro, juntamente com as outras fontes de apoio, pode contribuir para a adoção do papel materno de forma mais eficaz e segura.

Diante do exposto, este estudo objetivou investigar a atuação do profissional enfermeiro na rede de apoio social à gestante adolescente e sua influência no exercício do papel materno.

\section{Material e métodos}

Trata-se de uma pesquisa exploratória e descritiva, de abordagem qualitativa realizada em quatro Unidades de Atenção Primária à Saúde (UAPS), pertencentes à Secretaria Executiva Regional IV do município de Fortaleza, Ceará. O domicílio das gestantes adolescentes também se constituiu local de coleta de dados, em razão da baixa adesão destas às consultas de pré-natal.

A seleção dos locais de estudo se deu por amostragem não probabilística por conveniência. Participaram do estudo 16 gestantes adolescentes que faziam acompanhamento pré-natal nas UAPS, selecionadas segundo os critérios: a) inclusão: gestantes entre 12 e 18 anos de idade, segundo faixa etária padronizada pelo Estatuto da Criança e do Adolescente [10], cadastradas no SISPRENATAL, e que iniciaram o pré-natal independente do trimestre gestacional; b) exclusão: gestantes acompanhadas exclusivamente por profissional médico. Quanto aos enfermeiros, participaram do estudo 5 profissionais, selecionados segundo 0 tempo mínimo de seis meses de atuação na UAPS, o que formalizaria um certo vínculo com as gestantes.

A coleta de dados foi realizada no período de fevereiro a maio de 2014 por meio de duas técnicas: para a obtenção de informações das gestantes adolescentes, utilizou-se uma entrevista semiestruturada composta por questões relacionadas à identificação do perfil sociodemográfico e de sua rede de apoio; para avaliar a atuação do enfermeiro nas consulta de pré-natal, realizou-se a observação sistemática, a qual seguiu o seguinte roteiro: a) 0 enfermeiro identifica os aspectos psicossociais da gestante adolescente durante a consulta? b) $\mathrm{O}$ enfermeiro identifica a rede social de apoio à gestante? Se o faz, utiliza algum instrumento para tal? c) O enfermeiro permite que a gestante exponha suas dúvidas e as esclarece?

As entrevistas foram gravadas, transcritas na íntegra e organizadas em arquivos do Microsoft Word®, garantindo-se o sigilo e o anonimato das identidades das gestantes designando a cada uma o nome de uma pedra preciosa. Já a observação foi registrada no diário de campo do pesquisador e para cada enfermeiro atribuiu-se a letra $E$, seguida do número referente à ordem da observação.

Os dados foram analisados por meio da técnica de análise de conteúdo [11], focando-se particularmente na análise temática de conteúdo, em que o conceito central é o tema. Este comporta um feixe de relações e pode ser graficamente apresentado através de uma palavra, 
uma frase ou um resumo. Neste estudo, identificou-se a categoria $O$ apoio social da Atenção Primária à Saúde na maternidade adolescente.

A pesquisa foi aprovada pelo Comitê de Ética em Pesquisa da Universidade Estadual do Ceará, com parecer ํㅜ 681.375/2014 e pelas UAPS, por meio de anuência da Secretaria Municipal de Saúde de Fortaleza. Foram respeitados os aspectos éticos e legais preconizados pela Resolução 466/2012 do Conselho Nacional de Saúde [12]. Após explicação da pesquisa, os sujeitos atestaram sua concordância em participar da mesma mediante assinatura do Termo de Consentimento Livre e Esclarecido (TCLE). Nos casos de gestantes com menos de 18 anos, - TCLE foi assinado pelo responsável e as mesmas assinaram o Termo de Assentimento Informado.

Resultados

\section{Perfil das participantes}

As 16 participantes apresentaram idades entre 14 e 18 anos, todas primíparas, com tempo de estudo variando entre o $6^{\circ}$ ano do ensino fundamental até o ensino superior em curso, sete delas interromperam seus estudos antes ou durante a gestação. Nenhuma possuía vínculo empregatício e as rendas familiares variaram de $R \$ 356,00$ a $R \$ 3.000,00$. Em relação à religião, oito (50\%) afirmaram ser católicas, três (18,75\%) evangélicas, duas (12,50\%) cristãs protestantes e três $(18,75 \%)$ referiram não ter religião. Quanto ao relacionamento, nove $(56,25 \%)$ tinham união estável ou eram casadas e sete $(43,75 \%)$ eram solteiras.

\section{Categoria: O apoio social da Atenção Primária à Saúde na maternidade adolescente}

Esta categoria emergiu segundo a percepção das mães acerca do apoio advindo do profissional enfermeiro durante a consulta de pré-natal, além da captação do pesquisador por meio da observação sistemática.

O compromisso dos profissionais em realizar um bom atendimento e a orientação oferecida pela Equipe de Saúde da Família, especialmente pelos agentes comunitários de saúde (ACS), enfermeiros e médicos, foram enfatizados por algumas gestantes. O posto de saúde se constituiu em um local de apoio e suporte social, segundo as falas a seguir:

\footnotetext{
Eu gosto muito da agente de saúde porque ela se preocupa muito, vem sempre ver como eu estou, se estou bem, se tomei as vacinas. O restante é só no dia do pré-natal mesmo... Eles dizem pra eu comer bem, pra não fazer muito esforço, se eu sentir alguma coisa pra ir logo procurar ajuda. Essas coisas (Safira).
}

No entanto, para muitas das participantes, o apoio prestado pelo serviço de saúde se resumiu à oferta de uma consulta pré-natal puramente técnica focada na verificação de exames e avaliação do estado de saúde do bebê, sem aprofundar questões de cunho pessoal que avaliassem sua saúde psicológica e o meio social em que viviam. Quando questionadas sobre o que era mais importante durante a consulta de pré-natal, nenhuma das gestantes citou nada além do preenchimento do cartão de pré-natal, do exame físico abdominal e da prescrição medicamentosa. Compreende-se, assim, que muitas delas não perceberam a unidade de saúde como um dispositivo de apoio para além do cuidado biomédico.

Segundo o apreendido pelo pesquisador durante as consultas de pré-natal do enfermeiro, constatou-se que muito do que as gestantes relataram nas falas coadunavam com o observado. As consultas eram focadas basicamente na conferência dos exames apresentados pelas gestantes e, quando disponível, era feita a ausculta dos batimentos cardíacos fetais com sonar Doppler e a medição da altura uterina, garantindo a parte técnica da consulta. Raramente questões de abordagem psicossociais foram exploradas.

Diante do fato de que a maioria das gestantes em suas entrevistas não citou espontaneamente o enfermeiro como importante fonte de apoio, elas foram questionadas particularmente sobre o papel do enfermeiro nas consultas de pré-natal. As gestantes não souberam referir a importância destes profissionais no seu acompanhamento pré-natal.

Em diálogo com os enfermeiros envolvidos na pesquisa, pode-se perceber certa angustia dos mesmos, pois relataram a sobrecarga de trabalho como um importante fator impeditivo para uma consulta de pré-natal que levasse a um acompanhamento psicossocial aprofundado das gestantes, sem abordagens mais significativas neste aspecto. Em 
determinados momentos a enfermeira E1 e o enfermeiro E4 relataram que possuem tantas famílias pelas quais eram responsáveis em suas equipes de ESF que era impossível dar a atenção necessária que certos grupos requeriam, como no caso de gestantes adolescentes que precisam de um atendimento diferenciado dado o contexto vulnerável em que normalmente a gravidez acontece.

Uma observação relevante diz respeito ao baixo número de consultas de pré-natal realizadas pelas adolescentes, fato preocupante enfatizado pelos enfermeiros participantes da pesquisa. Estes relataram que as adolescentes realizavam de 3 a 5 consultas durante todo 0 período gestacional e expressaram dificuldade em atrair este público para as consultas. Retomando algumas fragilidades do serviço, os enfermeiros apontaram: elevada demanda, falta de tempo, falta de veículos para realizarem visita domiciliar, falta de interesse da própria equipe em formar grupo de gestante e criar vínculo, falta de interesse das gestantes que "não se importam muito" (E2) com suas gestações.

Outro aspecto negativo apresentado na fala da adolescente versa sobre o estigma social acerca da gravidez adolescente traduzido pela falta de acolhimento e juízo de valor demonstrado pela postura dos profissionais da UAPS:

[...] pro posto eu venho pro pré-natal porque é o jeito [...] pra mim, todo mundo aqui (Posto de Saúde) fica me olhando com cara de reprovação, me sinto um lixo. Eu sei que não se deve engravidar nova, que a gente precisa estudar e tal, mas aconteceu e eu não vou morrer por isso. Se eu aceitei essa gravidez e minha família também, então acho que as outras pessoas deveriam respeitar também e não ficar me julgando (Jade).

Este referido fato não foi percebido durante a observação da pesquisadora das consultas de pré-natal. Os enfermeiros tratavam as gestantes adolescentes com a mesma cordialidade que as demais. Contudo, quando as entrevistas eram realizadas nos domicílios das gestantes, percebeu-se que os ACS que acompanhavam a pesquisadora no campo, demonstravam nas entrelinhas certo preconceito em relação às adolescentes estarem grávidas, fazendo comentários negativos e moralistas.

No geral, as UAPS tiveram um papel bastante limitado como fonte de apoio citado pelas gestantes. Apenas três delas referiram este apoio como importante, sobretudo na figura do ACS. As demais mantinham relações enfraquecidas com as unidades, até mesmo relatando que não tinham interesse em frequentá-las e formar vínculo. Esse sentimento corroborou, inclusive, informações fornecidas pelos ACS que mencionaram que as gestantes deixavam de realizar suas consultas de pré-natal de forma regular e adequada pela falta do sentimento de pertença àquele espaço de cuidados.

Discussão

Estima que, anualmente, 7,6 milhões de adolescentes tornam-se mães. Destas, cerca de 70 mil morrem de causas relacionadas à gravidez e ao parto em países em desenvolvimento. Essas adolescentes tendem a originar-se de domicílios de baixa renda e a apresentarem deficiência nutricional [13].

$\mathrm{Na}$ realidade brasileira, evidenciada a partir de dados do Sistema de Informações sobre Nascidos Vivos (SINASC), percebe-se que a proporção de jovens entre 10 e 14 anos que tiveram filhos se manteve constante entre os anos de 2002 e 2013, destacando-se, nesse contexto, as regiões Norte e Nordeste do país [14].

As estatísticas relativas ao ano de 2013 mostram que dos 2.904 .027 nascidos vivos notificados ao SINASC, 559.991 (19,28\%) eram filhos de mães com idade até 19 anos, sendo $27.989(0,9 \%)$ de mães do grupo etário de 10 a 14 anos e $532.002(18,31 \%)$ de mães com idade de 15 a 19 anos [14]. Logo, percebe-se que jovens entre 15 e 19 anos se constituem na faixa etária que agrupa o maior número de adolescentes grávidas, o que está de acordo com os achados deste estudo, no qual das 16 gestantes adolescentes, quatorze estavam na faixa etária de 15 a 19 anos.

Estudo que entrevistou 4.634 jovens identificou que $29,6 \%$ das moças e $21,4 \%$ dos rapazes haviam experimentado a gravidez antes dos 20 anos. As jovens com renda familiar per capita de até $R \$ 220,00$, que engravidaram na adolescência, referiram mais frequentemente não terem concluído a educação básica. Entre os homens, ter renda familiar per capita até $\mathrm{R} \$ 220,00$, ter pais separados antes dos 20 anos e ter engravidado uma parceira antes dos 20 anos implica em maior chance de não concluir a educação básica [15]. 
A proporção de adolescentes com filhos é consideravelmente maior nos grupos de menor escolaridade. A incidência de gravidez é de uma em cada quatro mulheres para quem tem quatro anos de estudo ou menos (25\%). Em contraste, entre as mulheres com oito anos de estudo, uma em cada doze tem filhos (cerca de $8 \%$ ) [16].

A gravidez na adolescência é mais frequente nos estratos de renda mais baixa e, para muitas jovens, engravidar é uma escolha tomada como um meio de inserção social. É notável, ainda, a relação existente entre gravidez e abandono escolar, pois se estima que $57,8 \%$ das meninas brasileiras com filhos não estudam nem trabalham. Das meninas com idade entre $10 \mathrm{e}$ 17 anos sem filhos, somente $6,1 \%$ não estudavam; já entre as meninas com filhos, esta proporção chega a impressionantes $75,7 \%$, das quais $57,8 \%$ destas não estudavam nem trabalhavam, o que evidencia as dificuldades encontradas para engajar-se em alguma atividade fora de casa com um filho pequeno para cuidar [17]. Nesse sentido, há que se falar sobre a necessidade de articulação entre os setores de saúde e educação na busca de uma interdisciplinaridade capaz de enfrentar os desafios da orientação sexual para adolescentes [18].

Quanto à crença religiosa das participantes, referida em sua maioria como católica, corrobora a literatura [16]. Em estudo com 119.978 mulheres de 15 a 19 anos, 80,7\% se declararam católicas e $11,7 \%$ delas já tinham filhos. Os autores observaram os diferentes perfis de crenças religiosas e sua relação com a taxa de fecundidade, porém não chegaram a uma explicação concreta dessas variações. O que se pode destacar é que a espiritualidade tem papel importante no âmbito da maternidade adolescente. Os profissionais devem ter conhecimentos a respeito das crenças e valores das várias vertentes religiosas, além dos modos de vivenciar a espiritualidade, para estarem capacitados a desenvolver um cuidado significativo, fornecendo o apoio necessário nesse momento singular [19].

Quanto ao relacionamento, observou-se que a maior parte das participantes tinha um relacionamento estável, o que pode ser considerado um fator favorável, pois indica que a presença do companheiro pode funcionar como uma fonte de apoio neste contexto. Estudos apontam que a gestação na adolescência pode ser desejada e gratificante, promovendo o reconhecimento e a concretização de um projeto de vida, principalmente para aquelas com nível socioeconômico menos favorecido e que estejam em relações estáveis [20].

Pesquisas qualitativas apontam que muitas vezes tanto a moça quanto o rapaz planejam a gravidez, ou, pelo menos, não se esforçam muito para evitá-la e ficam contentes quando é confirmada. A adolescente busca construir sua identidade e sentir-se mais adulta, mais mulher e com mais poder tendo seu próprio filho. O projeto de vida profissional, neste caso, pode dar lugar ao de construir uma família ou, pelo contrário, o fato de ter um filho pode reforçar o plano de seguir estudando e buscar ascender socialmente [17].

A figura do companheiro como fonte de apoio social durante a gestação já é bem relatada na literatura. Estudo com adolescentes apontou que o companheiro ofertou carinho, amor e proferiu palavras que fortaleceram a autoestima da adolescente [21]. Outro estudo observou que as gestantes e puérperas receberam o apoio do companheiro/marido. Em relação ao papel de pai, gradualmente o homem tem atuado para além do suporte econômico da família, está se fazendo presente na alimentação e no cuidado com o bebê, inclusive no acompanhamento ao longo do seu desenvolvimento [22].

No contexto da maternidade adolescente, a equipe de saúde assume papel fundamental e deve estar preparada para enfrentar quaisquer fatores que possam afetar adversamente a gravidez, sejam eles clínicos, obstétricos ou de cunho socioeconômico ou emocional. Além do apoio informacional à gestante, orientando-a sobre o andamento de sua gestação e instruindo-a quanto aos comportamentos e atitudes que deve tomar para melhorar sua saúde, a equipe deve trabalhar no intuito de ampliar sua rede social de apoio, agregando família, companheiro(a) e pessoas de convivência próxima [23].

Estudo desenvolvido com puérperas, afirma que elas se sentiram acolhidas ao receberem dos profissionais de saúde informações com tranquilidade, destacando-as como únicas, conhecendo-as e auxiliando-as neste momento de mudanças e adaptações. Ao atender adolescentes, é fundamental ter dedicação e sensibilidade para perceber as demandas do período, bem como a necessidade de informação e orientação acerca dos direitos garantidos por lei, que podem auxiliá-las de maneira positiva [21].

Pesquisa "Nascer no Brasil", relacionada aos partos em primíparas adolescentes, evidenciou sua vulnerabilidade quando comparadas às mulheres adultas. As adolescentes apresentam mais desvantagens quanto aos seus direitos, com atraso escolar ou mesmo não 
frequentando a escola [24]. Faz-se necessário que os professores estejam mais próximos no período gravídico-puerperal e que atentem para as suas demandas [21].

Um aspecto preocupante ressaltado pelos profissionais de saúde se refere ao baixo número de consultas no pré-natal realizados pelas adolescentes. Estes enfatizaram que as gestantes adolescentes são as que mais faltavam às consultas de pré-natal, dificilmente perfazendo o mínimo de seis atendimentos preconizados pelo Ministério da Saúde [23].

O ACS, sempre que possível, deve intermediar as ações de saúde entre os profissionais do posto de saúde e a comunidade, com o intuito de promover vínculos e facilitar as relações interpessoais entre a comunidade e o serviço de saúde [25].

Sobre a competência da atenção básica, por meio da ESF, deve-se buscar proporcionar um ambiente acolhedor aos jovens, através de busca ativa e diálogo entre serviço de saúde/escola; oportunizar através das consultas com pais, familiares e responsáveis, informações pertinentes à saúde sexual dos jovens; discutir, ofertar e orientar sobre os métodos anticoncepcionais; proporcionar espaços resolutivos para o esclarecimento de dúvidas [26].

Em relação às situações de preconceito levantadas pelas gestantes, a literatura aponta que a desconstrução de preconceitos e estereótipos em torno da gravidez na adolescência significa pensar as relações de gênero em um contexto mais amplo de reflexão sobre a vivência da sexualidade na juventude. Contudo, isto deve ocorrer a partir de uma perspectiva renovada, que substitua o olhar moralizante, culpabilizador e amedrontador sobre os jovens pela valorização de seus direitos, entre eles os direitos sexuais e os direitos reprodutivos [17].

Apesar da maioria dos profissionais de saúde não valorizar aspectos psicossocioculturais, é possível realizar aproximações a uma abordagem integral e humanizada. Estas, praticadas por profissionais principalmente da saúde mental em atividades grupais junto às adolescentes gestantes, distinguem-se do modelo hegemônico da biomedicina pela presença ativa dos sujeitos nas trocas dialógicas e pela produção e desenvolvimento da autonomia das adolescentes gestantes [27].

No que concerne à satisfação de gestantes com a consulta de pré-natal realizada por enfermeiros, um estudo apontou resultados semelhantes, afirmando que é possível perceber, a partir dos relatos, que a satisfação refere-se apenas aos aspectos gerais. Um bom atendimento pré-natal, além de fazer um acompanhamento clínico da evolução gestacional, deve ser capaz de identificar intercorrências e prestar orientações quanto à alimentação, mudanças físicas e psicológicas durante o período gestacional. Percebe-se que não há um aprofundamento das consultas na tentativa de abordar questões mais densas [28].

Em estudo com puérperas adolescentes, contatou-se que os profissionais de saúde não foram mencionados como pessoas que as apoiavam, o que pode desvelar ausência de sensibilidade para perceber a subjetividade e as demandas das adolescentes em particular. A falta de apoio referida por algumas adolescentes acarretou abandono escolar, isolamento social e problemas de relacionamento com o companheiro e familiares. Estes fatos demonstram a necessidade de acompanhamento dos profissionais de saúde no ciclo gravídicopuerperal, para que possam atender às demandas das adolescentes e ampliar as suas possibilidades de fontes de apoio social [21].

O enfermeiro tem o compromisso, bem como a obrigação ética e moral, de envolver as famílias em seus cuidados de saúde [30]. Atividades de educação em saúde envolvendo gestantes e familiares, através de encontros e grupos, são recursos que permitem a aproximação entre profissionais e receptores do cuidado além de contribuírem para a assistência humanizada [28].

É neste sentido que o enfermeiro pode buscar superar a invisibilidade demonstrada pelo presente estudo, o qual aponta que muitas adolescentes sequer lembravam-se desse profissional como importante fonte de apoio para o desenvolvimento saudável de sua gestação.

Quando a gestante adolescente procura um profissional de saúde, este deve buscar investir na formação de vínculo para que estas adolescentes participem de grupos de apoio social através de um cuidado informal, passando a se ver em outras que estão vivenciando a mesma condição, ampliando desta forma sua rede de apoio, que influencia diretamente no desenvolvimento saudável da gestação. Estudo [2] afirma que a integração destas ações às atividades realizadas pelos serviços de saúde poderá efetivar-se nas UAPS a partir da identificação das famílias ou dos grupos sociais primários, não só como destinatários de ações assistenciais, mas também como copartícipes no processo de atenção à saúde. 
Neste estudo, a rede de apoio social contribuiu para as gestantes adolescentes no enfrentamento das adversidades inerentes ao seu processo adaptativo à nova realidade, sobretudo, considerando que todas eram primíparas e inexperientes ao vivenciar o papel materno.

Contudo, as relações enfraquecidas de apoio social ofertado pelos profissionais de saúde das UAPS foram pontos negativos observados tanto nas falas das gestantes adolescentes quanto na observação da pesquisadora durante as consultas de pré-natal.

Neste cenário, surge o questionamento: onde está a figura do enfermeiro e qual o seu real papel junto à comunidade? O que se percebe no presente estudo é que este profissional tem sua função fragilizada no que concerne ao apoio social oferecido à gestante para que esta desenvolva o seu papel materno. Com ações superficiais e empobrecidas de vínculos fortes é pouco provável que estes profissionais alcancem o objetivo de prestar uma assistência integral e de qualidade. Através dessa postura, acaba por manter um vínculo fraco com a comunidade, perpetuando um ciclo de ações pouco efetivas e frágeis, perdendo seu espaço como profissional de saúde da família.

Para estabelecer vínculos, faz-se necessário conhecer a família de fato: seus valores, suas percepções, suas dificuldades e suas forças na condução do cuidado cotidiano, pois só assim será possível uma assistência integral, mais eficaz e satisfatória do ponto de vista de quem a procura.

Ações de promoção da saúde e vínculo efetivo podem e devem ser realizadas por todas as pessoas que compõem as equipes multiprofissionais de saúde, pois estão aptos a prestar as devidas orientações com vistas a melhorar a qualidade de vida da população atendida. Estes devem buscar estratégias para que o vínculo seja formado, uma vez que as UAPS encontram-se inseridas na comunidade e tem como um de seus objetivos um maior e mais efetivo contato com a população.

Para traçar o perfil da rede de apoio social fornecida a uma gestante se faz necessário romper com as barreiras impostas pelo modelo biomédico centrado na doença e ainda ultrapassar os limites do individualismo que afastam as pessoas e dificultam as relações humanas, e buscar estratégias de aproximação tanto da gestante quanto da sua família e demais pessoas de seu convívio. Embasando-se neste conhecimento, pode-se propor mudanças adaptativas necessárias a essa nova etapa da sua vida. Portanto, é papel do enfermeiro ser parte integrante e fundamental dessa rede de apoio social, recuperando as redes de solidariedade primárias como provedoras de cuidados informais.

1. Brasil. Ministério da Saúde (BR). Pré-natal e puerpério. Atenção qualificada e humanizada. Brasília: MS; 2005.

2. Schwartz T, Vieira R, Geib LTC. Apoio social a gestantes adolescentes: desvelando percepções. Cienc Saude Colet 2011;16(5):2575-85. https://doi.org/10.1590/S141381232011000500028

3. Faquinello P, Carreira L, Marcon SS. A unidade básica de saúde a sua função na rede de apoio social ao hipertenso. Texto Contexto Enferm 2010;19(4):736-44. http://doi.org/10.1590/S0104-07072010000400017

4. Kim TH, Connolly JA, Tamim H. The effect of social support around pregnancy on postpartum depression among Canadian teen mothers and adult mothers in the maternity experiences survey. BMC Pregnancy Childbirth 2014;14(162):1-9. http://doi.org/10.1186/1471-2393-14-162

5. Pires R, Araújo-Pedrosa A, Canavarro MC. Examining the links between perceived impact of pregnancy, depressive symptoms, and quality of life during adolescent pregnancy: the buffering role of social support. Matern Child Health J 2014;18(4):789800 .

6. Leonidas C, Santos MA. Redes sociais significativas de mulheres com transtornos alimentares. Psicol: Reflex Crít 2013;26(3):561-71. https://doi.org/10.1590/S010279722013000300016

7. Rodrigues JZ, Oliveira PR, Ferreira JD, Batista DJR. O enfermeiro na atenção integral às mães adolescentes diante das transformações sociais e econômicas vivenciadas após o nascimento do filho. Revista Panorâmica 2014;16:19-31. 
8. Reis CC, Souza KRF, Alves DS, Tenório IM, Brandão Neto W. Percepção das mulheres sobre a experiência do primeiro parto: implicações para o cuidado de enfermagem. Ciencia e Enfermería 2017;23(2):45-56.

9. Brasil. Ministério da Saúde (MS). Secretaria Executiva. Departamento de Apoio à Descentralização. Diretrizes operacionais do pacto pela vida, em defesa do SUS e de gestão. Brasília: MS; 2006.

10. Brasil. Lei no 8.069, de 13 de julho de 1990. Dispõe sobre o Estatuto da Criança e do Adolescente e dá outras providências. Diário Oficial da União 1990; 27 ago.

11. Minayo MCS, Deslandes SF, Gomes R. Pesquisa Social: teoria, método e criatividade. 29 ed. Petrópolis: Vozes; 2010: p 79-108.

12. Brasil. Resolução $n^{\circ} 466$ de 12 de dezembro de 2012. Diretrizes e normas regulamentadoras para pesquisas envolvendo seres humano. Diário Oficial União 2013; 13 jun.

13. Fundo de População das Nações Unidas. Maternidade precoce: enfrentando o desafio da gravidez na adolescência. 2013:1-116.

14. DATASUS. Informações de Saúde. Estatísticas vitais 2015. [citado 2015 Jun 30]. Disponível:

http://www2.datasus.gov.br/DATASUS/index.php?area=0205\&VObj=http://tabnet.datas us.gov.br/cgi/deftohtm.exe?sinasc/cnv/nv.

15. Almeida MCC, Aquino EML. Adolescent pregnancy and completion of basic education: a study of young people in three state capital cities in Brazil. Cad Saúde Pública 2011;27(12):2386-400. https://doi.org/10.1590/S0102-311X2011001200010

16. Longo L, Ribeiro PM. Fecundidade e Fé [Internet]. Universidade Federal de Minas Gerais 2010. [citado 2014 Maio 31]. Disponível em: https://www.ufmg.br/boletim/bol1692/6.shtml.

17. Fontoura NO, Pinheiro LS. Gravidez na adolescência. Instituto de Pesquisa Econômica Aplicada (IPEA). Desafios do desenvolvimento 2010; 60:29.

18. Mendes SS, Moreira RMF, Martins CBG, Souza SPS, Matos KF. Saberes e atitudes dos adolescentes frente à contracepção. Rev Paul Pediatr 2011;29(3):385-91. https://doi.org/10.1590/S0103-05822011000300013

19. Hoga LAK, Borges ALV, Reberte, LM. Razões e reflexos da gravidez na adolescência: narrativas dos membros da família. Esc Anna Nery Rev Enferm 2010;14(1):151-57. https://doi.org/10.1590/S1414-81452010000100022

20. Caminha ON, Freitas LV, Herculano MMS, Damasceno AKC. Pregnancy in adolescence: from planning to the desire to become pregnant - descriptive study. Online Braz J Nurs 2010;9(1). https://doi.org/10.5935/1676-4285.20102872

21. Cremonese L, Wihelm LA, Prates, LA, Paula CC, Sehnem GD, Ressel LB. Apoio social na perspectiva da puérpera adolescente. Esc Anna Nery Rev Enferm 2017;21 (4):e20170088. http://doi.org/10.1590/2177-9465-ean-2017-0088

22. Ribeiro JP, Gomes GC, Silva BT, Cardoso LS, Silva PA, Strefling ISS. Participação do pai na gestação, parto e puerpério: refletindo as interfaces da assistência de enfermagem. Espaç Saúde 2015;16(3):73-82. https://doi.org/10.22421/1517$7130.2015 \mathrm{v} 16 \mathrm{n} 3 \mathrm{p} 73$

23. Brasil. Ministério da Saúde (MS). Secretaria de Atenção à Saúde. Departamento de Atenção Básica. Atenção ao pré-natal de baixo risco. Brasília: MS; 2012.

24. Gama SGN, Viellas EF, Schilithz AOC, Theme Filha MM, Carvalho ML, Gomes KRO et al. Factors associated with caesarean section among primiparous adolescentes in Brazil, 2011-2012. Cad Saúde Pública 2014;30(Supl. 1):S117-S27. https://doi.org/10.1590/0102-311X00145513

25. Vieira SM, Bock LF, Zocche DA, Pessota CU. Percepção das puérperas sobre a assistência prestada pela equipe de saúde no pré-natal. Texto Contexto Enferm 2011;20(n. especial):255-62. https://doi.org/10.1590/S0104-07072011000500032

26. Spindola T, Ribeiro KS, Rodrigues V, Fontes F. A vivência da gravidez na adolescência: contribuições para a enfermagem obstétrica. Adolesc Saude 2015;12(1):50-6.

27. Santos MMAS, Saunders C, Baiao MR. A relação interpessoal entre profissionais de saúde e adolescente gestante: distanciamentos e aproximações de uma prática integral e humanizada. Cienc Saude Colet 2012;17(3):775-86. https://doi.org/10.1590/s141381232012000300025

28. Santos AL, Radovanovic CAT, Marcon SS. Assistência pré-natal: satisfação e expectativas. Rev RENE 2010;11(S):61-71. 
29. Wrigth LM, Leahey M. Enfermeiras e famílias: um guia para avaliação e intervenção na família. 5 ed. São Paulo: Roca; 2012. 